\title{
Numerical Modeling of 2-D Conductive Heat Transfer and Its Application for the Characterization of Geothermal Systems
}

\author{
Setya Drana Harry Putra ${ }^{1}$, Silvia Jannatul Fajar ${ }^{2}$, Wahyu Srigutomo ${ }^{3}$ \\ ${ }^{1}$ Geothermal Engineering, Faculty of Mining and Petroleum Engineering, Bandung Institute of Technology \\ ${ }^{2}$ Applied Geophysics, Faculty of Mining and Petroleum Engineering, Bandung Institute of Technology \\ ${ }^{3}$ Physics of Earth and Complex Systems, Faculty of Mathematics and Natural Sciences, Bandung Institute of Technology \\ Jalan Ganeca 10, Bandung, West Java, Indonesia \\ 1e-mail: setya.drana@hotmail.com
}

\begin{abstract}
A geothermal system is basically a system where heat is transferred from the internal part to the surface of the earth dominantly by conduction, convection, or both. The spatial variation of the magnitude of conductive heat transfer represented by lateral variation of observed surface heat flow values is heavily related to the subsurface temperature distribution, the pattern of which is directly controlled by the variation of rock thermal conductivity values. Therefore, the information about subsurface temperature distribution may provide insight for the interpretation of the thermal structure of a region, within a more regional framework of geothermal systems in particular. In this research, we performed a numerical forward modeling procedure of 2-D conductive heat transfer using finite difference solution of the steady state heat conduction equation via a Gauss-Seidel scheme. The main physical parameters used as the input in the modeling procedure are rock thermal conductivity values as well as temperature boundary conditions. The modeling scheme was applied on two different synthetic common geothermal system geometries, one within a volcanic setting and one within a sedimentary environment, by using appropriate thermal conductivities and temperature boundary conditions for the assumed lithologies within each respective setting. The results of the modeling procedure were able to effectively characterize the thermal structure and surface heat flow patterns of the geothermal system in both environments.
\end{abstract}

Keywords: geothermal system, conductive heat transfer, surface heat flow, numerical modeling, finite difference method

\section{INTRODUCTION}

Geothermal systems are essentially the representations of natural mechanisms for which heat is transferred from the hotter, inner part to the cooler surface of The Earth [9]. In general there are three different mechanisms by which heat can be transferred, namely conduction, convection, and radiation [4]. However, within the earth the dominant mode of heat transfer is conduction through solid rocks, while convection only plays a significant role in areas with extensive magmatic and hydrothermal activities such as active volcanic arcs as well as some tectonically active regions [10], where heat is brought to the surface by magma in volcanic eruptions, circulating groundwater, and/or uplift and erosion [17]. On the contrary, radiation is the primary mechanism where heat is delivered from the Sun to the surface of The Earth and therefore is not of great relevance to geothermal systems, where the heat source is located inside The Earth.

The portion of Earth's heat transferred via conduction is proportional to the product of temperature difference between the surface and the subsurface at a particular depth and the thermal conductivity of the rocks through which heat is passing. The relevant physical quantity for this fraction of heat is called the terrestrial heat flow [2], which can be observed directly at the surface by drilling to a depth and measuring temperature between the surface and that depth as well as the thermal conductivity of the materials that were drilled into [1]. The spatial variation of the magnitude of heat flow provides an indication of the variation in the subsurface temperature distribution, the pattern of which in turn is directly controlled by the variation of rock thermal conductivity values as well as depths to heat sources and the presence of a geothermal system.

Unfortunately, current drilling campaigns are limited to depths of only a few kilometers beneath the Earth and at certain places, rendering direct and complete characterization of the entire subsurface thermal regime that may be related to the presence of geothermal systems impossible to be carried out. Therefore it is necessary to predict these parameters using alternative ways, one of which is via the method of numerical modeling [16; 18]. It is worth noting that numerical simulations of geothermal systems are primarily focused on the behavior of the reservoir element of the system in question for engineering purposes (e.g. [19]) rather than on its entire thermal structure. Thus in this research, we performed numerical forward modeling of 2-D conductive steady state temperature distribution similar to previous work done by authors such as [6] and [14] to characterize the overall thermal regime of geothermal systems. The work was based on synthetic geological models representing two common environments that are known to host geothermal systems, namely volcanic [12] and sedimentary basin [3] settings. The results of the numerical modeling were then used as an aid to interpreting the thermal structure as well as causes of possible observed patterns of heat flow anomalies associated with each of these settings. 


\section{METHODOLOGY}

\section{A. Governing Equation and Finite Difference Approximation}

In order to characterize the process of heat transfer in geothermal systems it is necessary to first understand the underlying physical principle. The general equation governing heat transfer mechanisms in The Earth is the heat equation, which can be written as follows $[11 ; 7]$ :

$$
\frac{d\left[\rho_{b} C_{b} T\right]}{d t}=\lambda\left(\frac{d^{2} T}{d x^{2}}+\frac{d^{2} T}{d y^{2}}+\frac{d^{2} T}{d z^{2}}\right)-V \rho C\left(\frac{d T}{d x}+\frac{d T}{d y}+\frac{d T}{d z}\right)+A
$$

Where $\rho_{b}, C_{b}$ and $\rho, C$ each is the bulk rock and fluid density $\left(\mathrm{kg} \cdot \mathrm{m}^{-3}\right)$, bulk rock and fluid specific heat capacity $\left(\mathrm{kJ} \cdot \mathrm{kg}^{-1}\right)$, $\lambda$ is the rock thermal conductivity $\left(\mathrm{W} \cdot \mathrm{m}^{-1} \cdot \mathrm{K}^{-1}\right), V$ is the fluid velocity $\left(\mathrm{m} . \mathrm{s}^{-1}\right)$, and $A$ is the rock internal heat production $\left(\mathrm{W} . \mathrm{m}^{-3}\right)$. The term on the left hand side of the equation is usually referred as the transient term, whereas the first, second, and third terms on the right hand side are called diffusive, advective, and source terms, respectively. The physical meaning of (1) is that the change of temperature over a control volume can be introduced by the difference of the heat fluxes between both ends of that volume, the redistribution of heat by fluid movement, the internal heat generation of the volume, or all of the three processes. In this research we only consider the mechanism of heat transfer by conduction, so that (1) can be simplified to:

$$
\frac{d\left[\rho_{b} C_{b} T\right]}{d t}=\lambda\left(\frac{d^{2} T}{d x^{2}}+\frac{d^{2} T}{d y^{2}}+\frac{d^{2} T}{d z^{2}}\right)+A
$$

By further assuming that the heat transfer process is in steady state and that the source term can be neglected due to its relatively small importance, (2) further reduces to the steady state heat conduction equation:

$$
\lambda\left(\frac{d^{2} T}{d x^{2}}+\frac{d^{2} T}{d y^{2}}+\frac{d^{2} T}{d z^{2}}\right)=0
$$

So that,

$$
\nabla^{2} T=0
$$

Which is the Laplace's equation, as mentioned in [13]. We utilized a discretized version of (3) in order to be able to put it into a numerical forward modeling scheme by replacing the continuous derivative with a difference equation using the so-called finite difference approximation [4]. The 2-D finite difference formulation of (3) is as follows [18]:

$$
\begin{aligned}
0 & =\left(\frac{\left(\frac{\lambda_{i+1, j}+\lambda_{i, j}}{2}\right)\left(\frac{T_{i+1, j}-T_{i, j}}{\Delta x}\right)-\left(\frac{\lambda_{i, j}+\lambda_{i-1, j}}{2}\right)\left(\frac{T_{i, j}-T_{i-1, j}}{\Delta x}\right)}{\Delta x}\right) \\
& +\left(\frac{\left(\frac{\lambda_{i, j+1}+\lambda_{i, j}}{2}\right)\left(\frac{T_{i, j+1}-T_{i, j}}{\Delta y}\right)-\left(\frac{\lambda_{i, j}+\lambda_{i, j-1}}{2}\right)\left(\frac{T_{i, j}-T_{i, j-1}}{\Delta y}\right)}{\Delta y}\right)
\end{aligned}
$$

Which, to solve for the temperature at a particular node $\left(T_{i, j}\right)$, becomes:

$$
\begin{array}{r}
T_{i, j}=\frac{\left(\frac{\lambda_{i+1, j} T_{i+1, j}+\lambda_{i, j} T_{i+1, j}+\lambda_{i, j} T_{i-1, j}+\lambda_{i-1, j} T_{i-1, j}}{2 \Delta x^{2}}\right)}{\left(\left(\frac{\lambda_{i+1, j}+2 \lambda_{i, j}+\lambda_{i-1, j}}{2 \Delta x^{2}}\right)+\left(\frac{\lambda_{i, j+1}+2 \lambda_{i, j}+\lambda_{i, j-1}}{2 \Delta y^{2}}\right)\right)} \\
+\frac{\left(\frac{\lambda_{i, j+1} T_{i, j+1}+\lambda_{i, j} T_{i, j+1}+\lambda_{i, j} T_{i, j-1}+\lambda_{i, j-1} T_{i, j-1}}{2 \Delta y^{2}}\right)}{\left(\left(\frac{\lambda_{i+1, j}+2 \lambda_{i, j}+\lambda_{i-1, j}}{2 \Delta x^{2}}\right)+\left(\frac{\lambda_{i, j+1}+2 \lambda_{i, j}+\lambda_{i, j-1}}{2 \Delta y^{2}}\right)\right)}
\end{array}
$$

\section{B. Synthetic Geological Models, Grid Node Geometry and Boundary Conditions}

The finite difference approximation (5) was applied on two pairs of geological synthetic cases of real geothermal systems, one hosted in a volcanic setting [12] and one in a sedimentary basin setting [3]. Each of the cases consists of a thermal conductivity and an initial temperature distribution models (Figure 1 and 2). These geological models were then used as input variables for the numerical modeling procedure [8].

The values of rock thermal conductivity were taken from available literature (e.g. $[1 ; 11]$ ). The initial temperature distribution was calculated by extrapolating to depth the temperature from a constant value-type surface boundary condition of $25{ }^{\circ} \mathrm{C}$ with a geothermal gradient value of 30 ${ }^{0} \mathrm{C} / \mathrm{km}$ (except for nodes occupied by a heat source of fixed temperature). The model domains are composed of 25 rows x 50 columns of grid nodes, the spacing of each which was taken to be 200 meters, yielding a total model dimension of $5000 \times 10000$ meters. For the two models, the lateral boundary conditions are set to zero heat flow value by enforcing zero thermal conductivity at the respective boundary cells. We adopted a Gauss-Seidel scheme [5] to iteratively solve (6) for the temperature value at each grid node. The method was selected due to it being simpler to code and implement, while achieving convergence as equally fast as other linear iterative solvers.

The volcanic model pair is based on the replica of a stratovolcano which is assumed to be devoid of hydrothermal activities. The model geometries and initial temperature distributions are shown in Figure 1, with the difference between the two models lies only in the geometry and dimension of the heat source. For Model 1 (Figure 1.A), the cooling magmatic heat source is located beneath the center of the volcanic caldera at a depth of $4000 \mathrm{~m}$, the shape of which more or less follows the topography of the volcanic edifice. Model 2 (Figure 1.B) displays a heat source distribution that is much more laterally extensive but is located at a higher depth $(4400 \mathrm{~m})$. The temperature of magmatic heat source is assumed to be $600{ }^{0} \mathrm{C}$ to simulate a more felsic intrusion. The basal boundary nodes are set to a constant value taken from 
the initial temperature distribution, i.e. bottom grid nodes that are not occupied by heat source. The rock materials are composed of lava, pyroclastics, and the sedimentary basement rocks. Thermal conductivity values of these rocks are listed in Table 1.

(A)

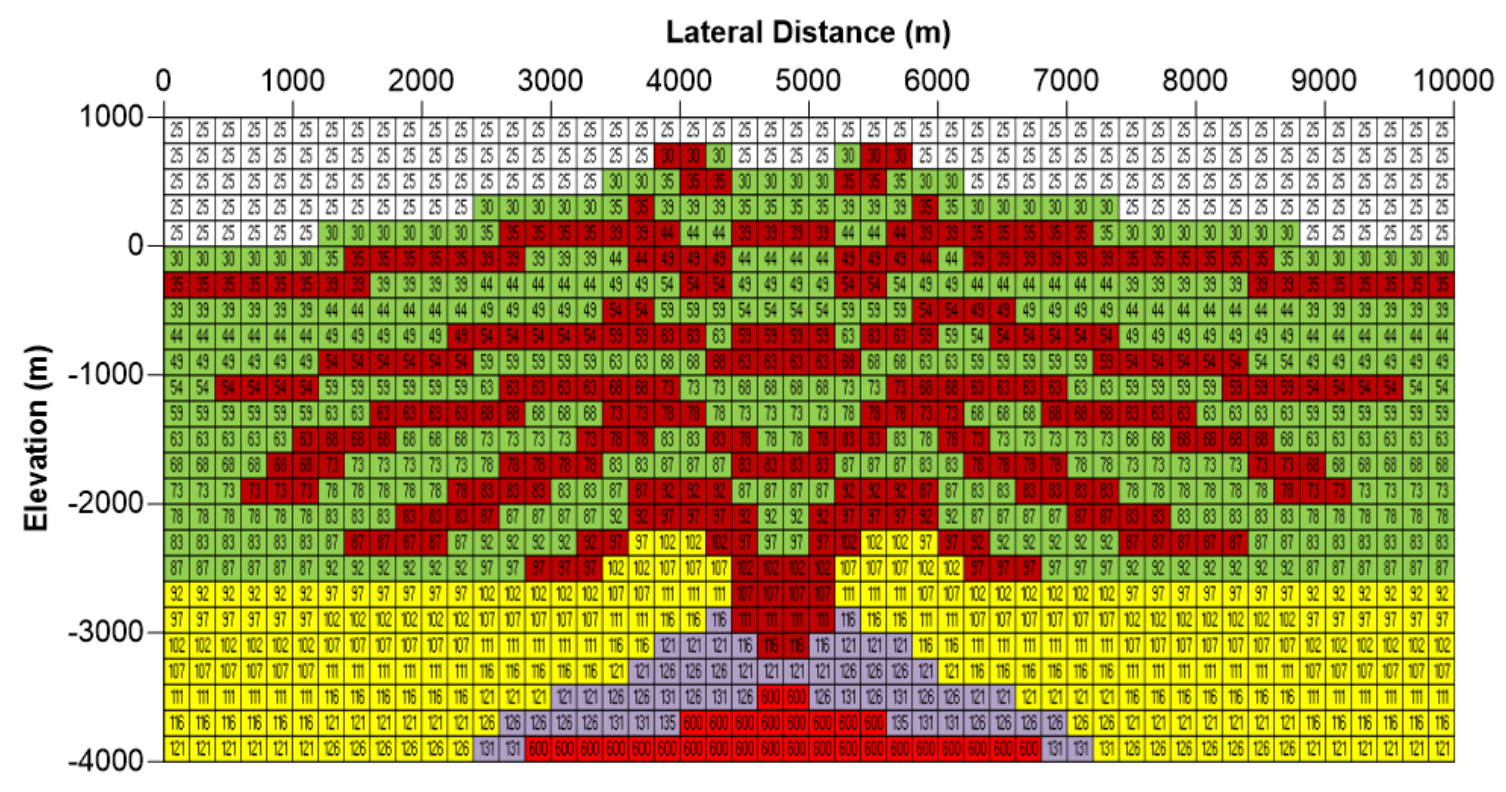

(B)

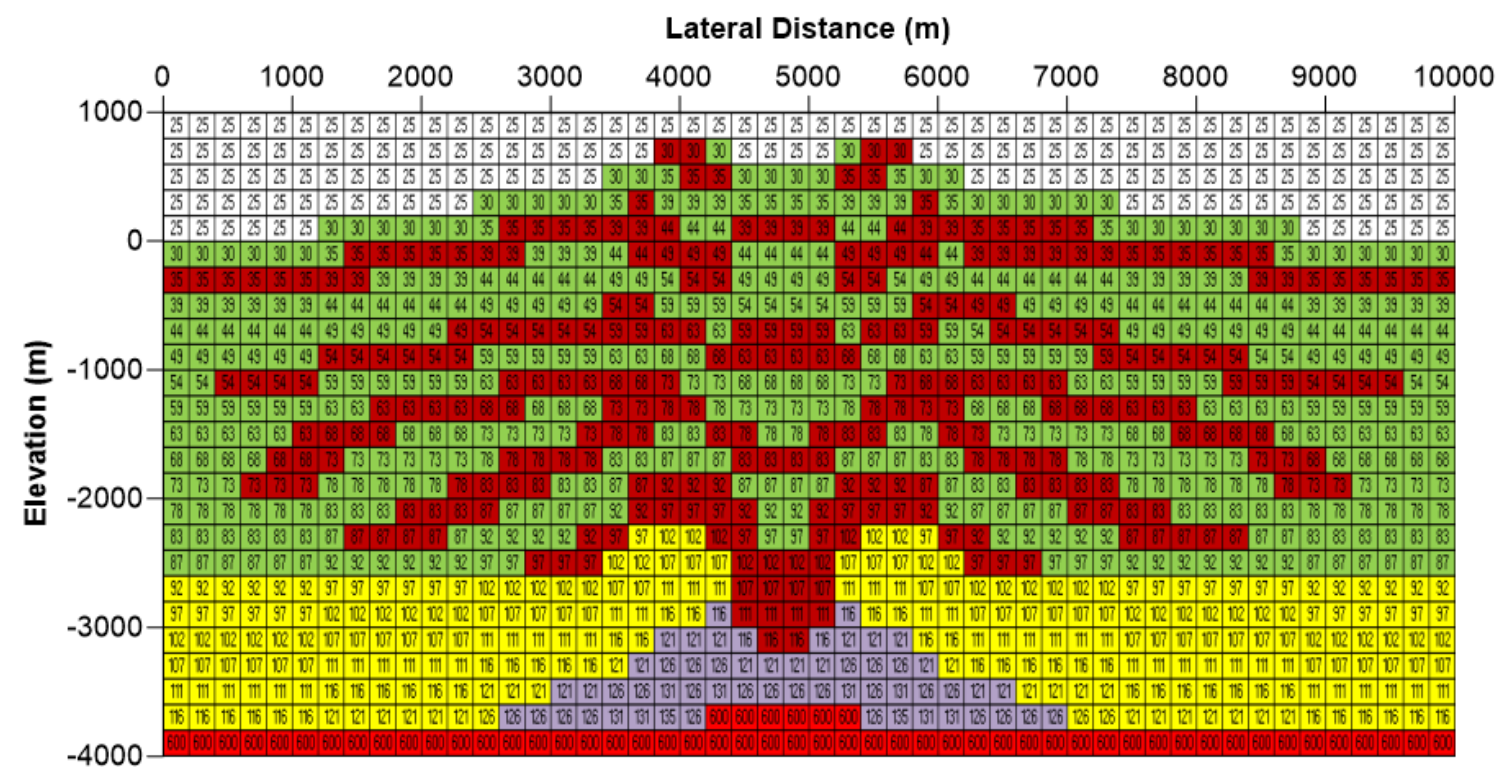

Figure 1. Geometry of the synthetic stratovolcano model pair: Model 1 (A) and Model 2 (B). Values within the cells correspond to the initial temperature for each of the respective grid nodes. 
TABLE 1 . Thermal conductivity values of the rock types used in the volcanic models.

\begin{tabular}{|l|c|c|}
\hline \multirow{2}{*}{$\begin{array}{c}\text { Color } \\
\text { Index }\end{array}$} & Rock Type & $\begin{array}{c}|c| \\
\text { Thermal } \\
\text { Conductivity } \\
\left(\mathbf{W m}^{-1} \mathbf{K}^{-1} \text { ) }\right.\end{array}$ \\
\cline { 2 - 3 } & $\begin{array}{c}\text { Felsic Magma (Heat } \\
\text { source) }\end{array}$ & 2.0 \\
\hline \multirow{2}{*}{} & $\begin{array}{c}\text { Contact Metamorphic } \\
\text { Rock }\end{array}$ & 2.7 \\
\hline & $\begin{array}{c}\text { Sandstone (Basement) } \\
\text { Pyroclastic Rock }\end{array}$ \\
\hline & Lava & 2.3 \\
\hline & $\begin{array}{c}\text { Air (surface } \\
\text { topography) }\end{array}$ & 2.6 \\
\hline
\end{tabular}

The basin model is based on an extensional sedimentary basin where heat transfer by conduction dominates and the effect of advective transfer of heat by circulating groundwater is negligible due to flat surface topography. The model geometry is shown in Figure 2. The basal boundary condition is set to constant temperature of $200{ }^{\circ} \mathrm{C}$ at depths. For Model 1 (Figure 2.A), the overall geometry of this constant temperature layer follows the basement rock configuration to some degree, while for Model 2 (Figure 2.B) the layer is located at a fixed depth of $4400 \mathrm{~m}$. The rock types and their corresponding thermal conductivity values included in the models are listed in Table 2.

TABLE 2. Thermal conductivity values of the rock types used in the sedimentary basin models.

\begin{tabular}{|c|c|c|}
\hline \multirow{2}{*}{$\begin{array}{c}\text { Color } \\
\text { Index }\end{array}$} & Rock Type & $\begin{array}{c}\text { Thermal } \\
\text { Conductivity } \\
\left(\mathbf{W m}^{-1} \mathbf{K}^{-1}\right)\end{array}$ \\
\cline { 2 - 3 } & Granite (Basement) & 3.5 \\
\hline & Sandstone & 3.2 \\
\hline & Shale & 1.5 \\
\hline & Shaly Sandstone (1:1) & 2.3 \\
\hline & Limestone & 2.7 \\
\hline & Air (Surface topography) & 0.0 \\
\hline
\end{tabular}

\section{RESULTS AND DISCUSSION}

\section{A. Sensitivity Study}

We conducted a sensitivity study of the effect of model parameters, geometry, and boundary conditions to the proposed numerical algorithm prior to implementation on the synthetic geological model by utilizing simple models. The first model consists of a single rectangular box embedded in a medium with a different thermal conductivity (Figure 3.A and 3.B), while the second one consists of two rectangular boxes located at the edges of the model domain (Figure 4.A and 4.B). The thermal conductivity values assigned for the boxes as well as the medium are 1.5 and 5.0 $\mathrm{W} \cdot \mathrm{m}^{-1} \cdot \mathrm{K}^{-1}$. The model space dimension is similar to the previous geological synthetic models. The initial temperature distribution was assumed to be zero at every node except the boundary nodes. A zero lateral heat flow was imposed on each lateral side, with $0^{\circ} \mathrm{C}$ and $500^{\circ} \mathrm{C}$ constant surface and basal temperatures.

The result of simulation for the single rectangular box model shows that the temperature field is distorted near the center of the model space for the two cases of thermal conductivity contrasts (Figure 5.A and 5.B). This suggests that the algorithm was able to resolve the presence of a single thermal conductivity anomaly that is represented by the pattern of temperature distribution. In contrast, the result of numerical simulation using the two-rectangular box model shows that the two temperature distributions follow a somewhat rather indistinguishable pattern (Figure 6.A and 6.B). This was interpreted as having resulted from the effect of the imposed lateral boundary conditions, rather than the geometry of the thermal conductivity anomaly itself. It suggests that the presence of thermal conductivity anomalies can not be resolved well when they are situated near the boundary of the model space.

\section{B. Numerical Modeling using Synthetic Geological Models}

Based on the results of the previous sensitivity study, it was decided to enlarge the horizontal dimension of the model space up to three times of its original extent to reduce the influence of lateral boundary condition on the modeled temperature near the lateral sides [15]. This yielded a model space dimension of $5000 \times 30000$ meters $(25 \times 152$ grid nodes) in size. All numerical computations were carried out with MATLAB software using a $2.4 \mathrm{GHz}$ Intel Core i73630QM-based CPU with $4.0 \mathrm{~GB}$ amount of RAM and a 2.0 GB NVIDIA GEFORCE® GT 650M Graphics Processing Unit. The following results presented were obtained after 200 iterations. Each computation takes approximately 84.67 seconds to perform. 
(A)

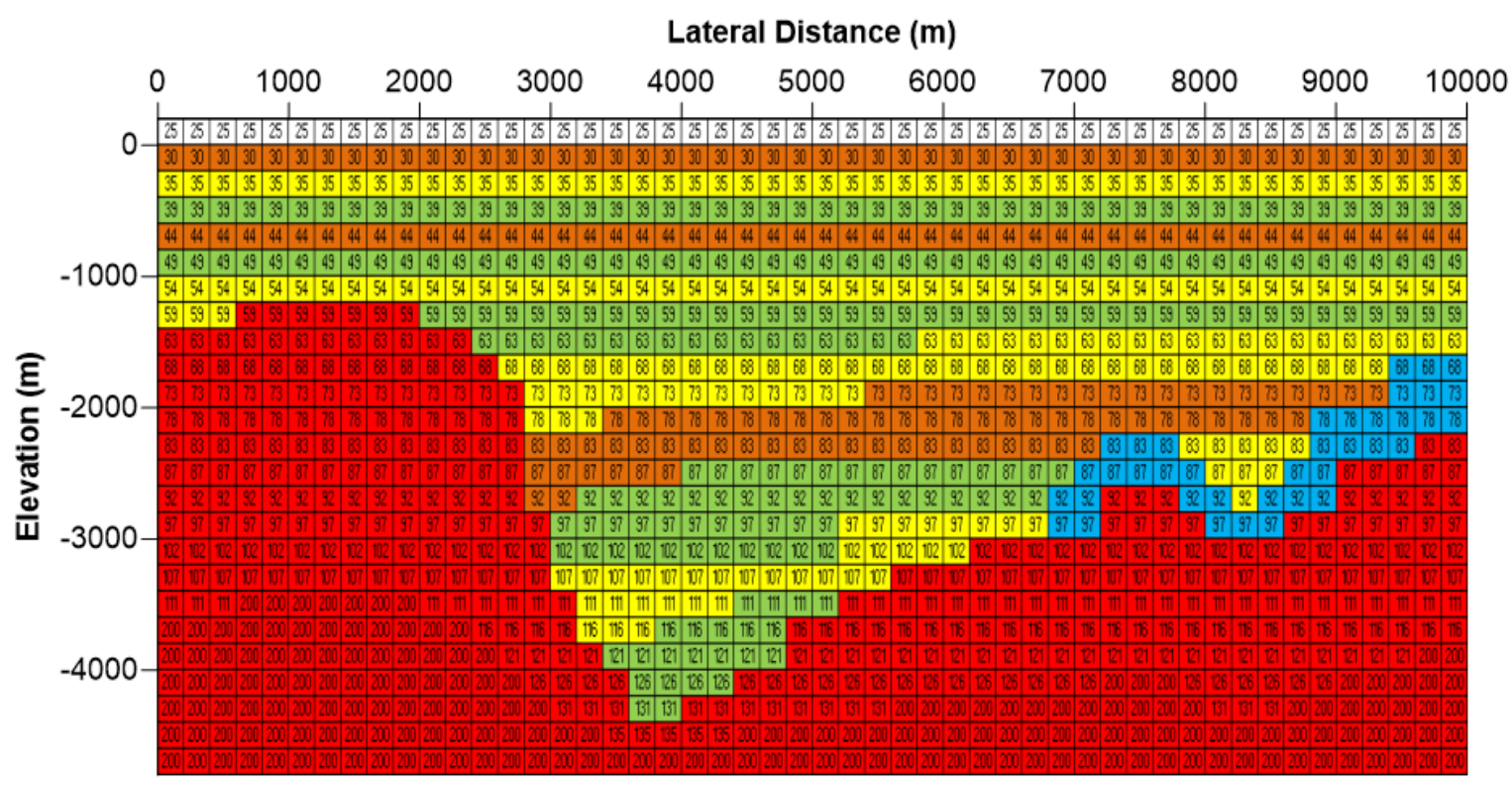

(B)

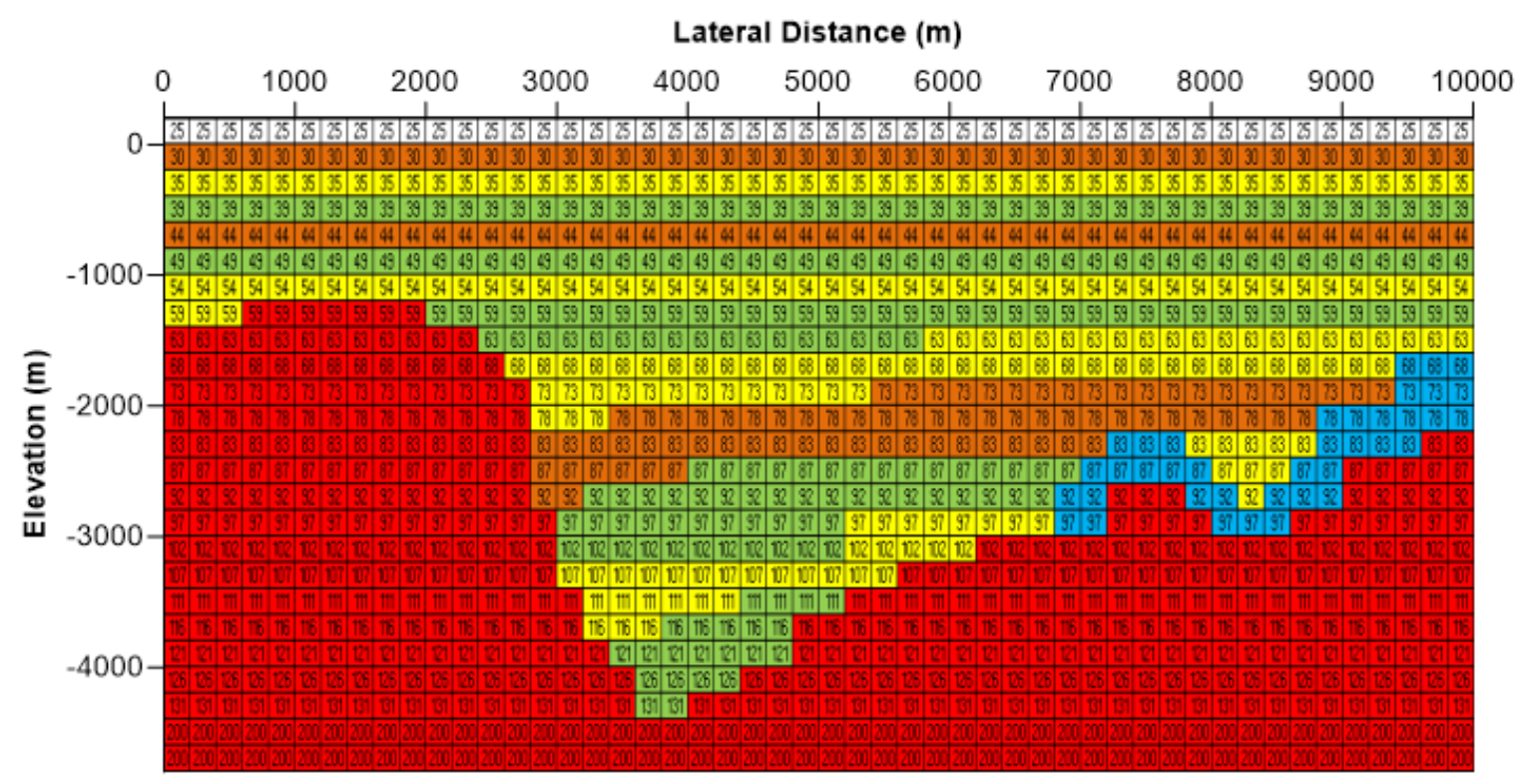

Figure 2. Geometry of the synthetic extensional sedimentary basin model pair: Model 1 (A) and Model 2 (B). Values within the cells correspond to the initial temperature for each of the respective grid nodes.

Figure 7.A shows the final result of the numerical modeling of 2-D steady state conductive temperature distribution for the synthetic volcanic Model 1. The overall pattern of modeled temperature structure shows a similarity with the shape of the magmatic intrusion. This is quite logical since we did not include, in our synthetic model, other types of heat sources at any other locations. It can also be argued that this behavior could have in some part resulted from the structural pattern of thermal conductivity itself that is strongly layered in most part of the volcano's flanks. The residual temperature, obtained from calculated values of the last iteration minus the values from previous iteration, is given in (Figure 7.B). It also displays a distribution which nearly resembles the geometry of magmatic intrusion, with 
the highest values concentrated just above the intrusive body. This was interpreted as having resulted from the intrusion's location, because the temperature values at grid nodes located at close proximity to the heat sources are the first that experienced the highest modification in each subsequent iteration. This is further enhanced by the geometrical relationship between the rock layers beneath the volcano's caldera and the rock layers beneath its flanks, which we intentionally created so as to represent a lateral discontinuity in the model.

(A)

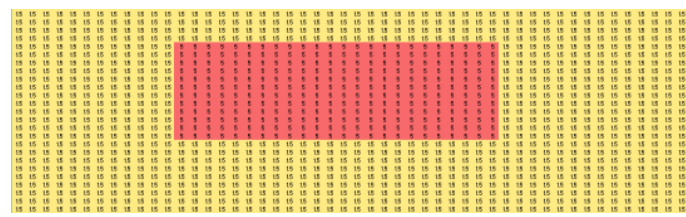

(B)

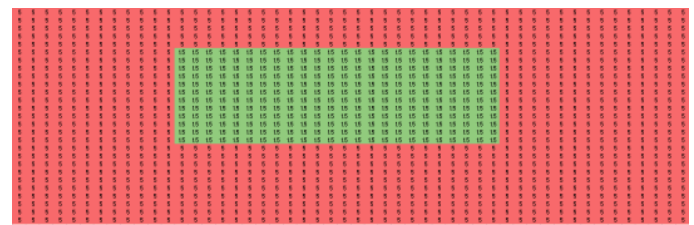

Figure 3. Single rectangular box model used for sensitivity study where the thermal conductivity of the box is (A) higher (red) and (B) lower (green) than the thermal conductivity of the surrounding medium.

(A)

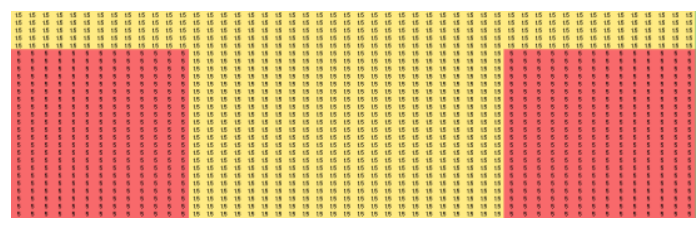

(B)

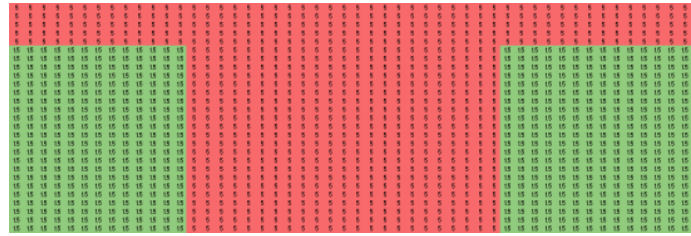

Figure 4. Two rectangular box model used for sensitivity study where the thermal conductivities of the boxes are (A) higher (red) and (B) lower (green) than the thermal conductivity of the surrounding medium.

(A)

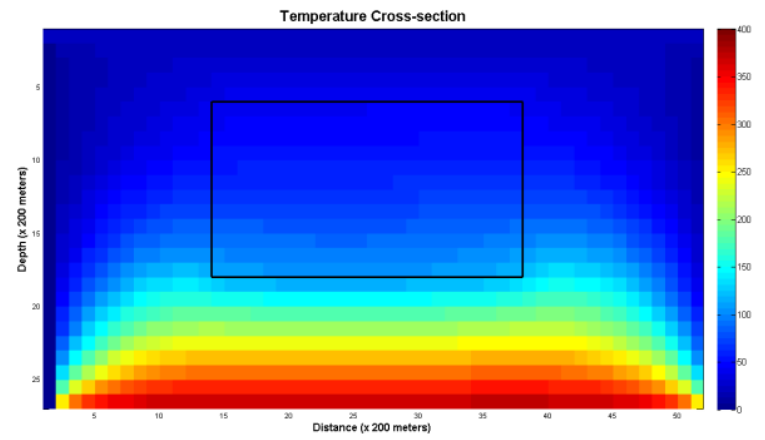

(B)

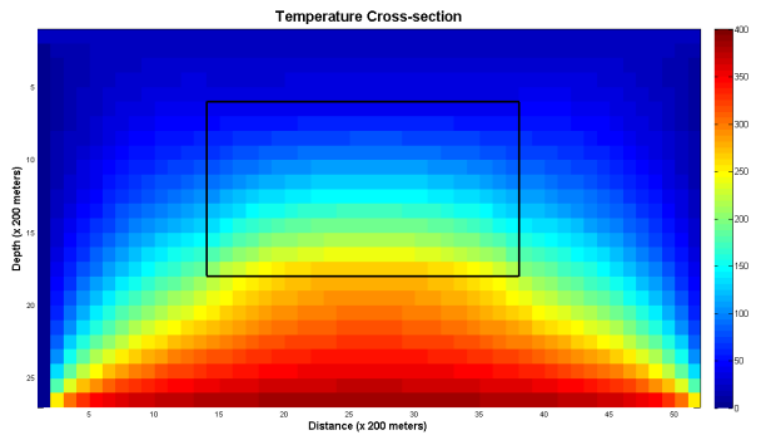

Figure 5. Results of the numerical simulation of temperature distribution (in ${ }^{0} \mathrm{C}$ ) for the single rectangular box model of (A) Figure 3.A and (B) Figure 3.B (after 200 iterations). The position of the box is given by the black rectangle superimposed on the simulation result.

(A)

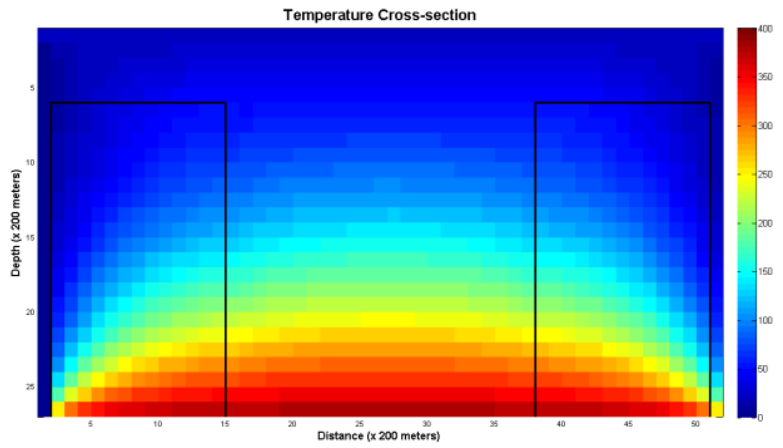

(B)

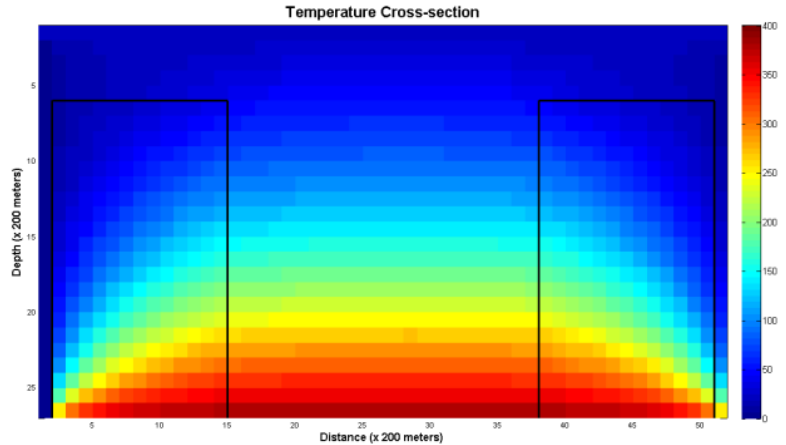

Figure 6. Results of the numerical simulation of temperature distribution (in ${ }^{0} \mathrm{C}$ ) for the two-rectangular box model of (A) Figure 4.A and (B) Figure 4.B (after 200 iterations). The positions of the boxes are given by the black rectangles superimposed on the simulation result.

In addition, we calculated the surface heat flow by multiplying the temperature gradient with the arithmetic mean of thermal conductivity values at a particular lateral grid position (Figure 7.C). This procedure results in a higher estimate of heat flow values as opposed to using the harmonic mean of thermal conductivity [1], but can be justified as we only desired to analyze their pattern and differences rather than absolute values. It can be observed that the heat flow profile over a volcanic caldera is extremely elevated relative to its flanks, the extent of which defines the lateral boundary of the causative magmatic heat source. The maxima of this anomaly is rather flat-topped 
than forming a single prominent peak resembling the overall shape of the heat source, which provides an indication that the volcano's topography may also be involved to an extent in dictating the shape of the observed heat flow anomaly.

The result of numerical modeling based on the second volcanic model in Figure 8 further explains the extent of relative proportions of influence between the geometry of magmatic heat source and the rock thermal conductivity structure on the modeled temperature distribution. While the distribution of the residuals confirms the previous explanation for the observed residual pattern, it can be seen that the temperature structure does not strongly obey the pattern of thermal conductivity structure, suggesting that heat source geometry poses a much stronger influence.
In contrast, the surface heat flow profile displays an inverted shape as compared to the one based on previous volcanic model. A combination of thermal conductivity distribution and topographical effect is a possible cause to this observed behavior. The average thermal conductivity values beneath the flanks of the volcano are increased relative to the values beneath the caldera due to the presence of thick sedimentary basement rocks, whereas the distance between the caldera floor and the heat source becomes the longest, thus elevating the surface heat flow values over the flanks relative to the caldera. This has convinced us that topography and thermal conductivity structure have a more profound impact on the observed heat flow anomaly than does the overall geometry of the heat source for this model.

(A)

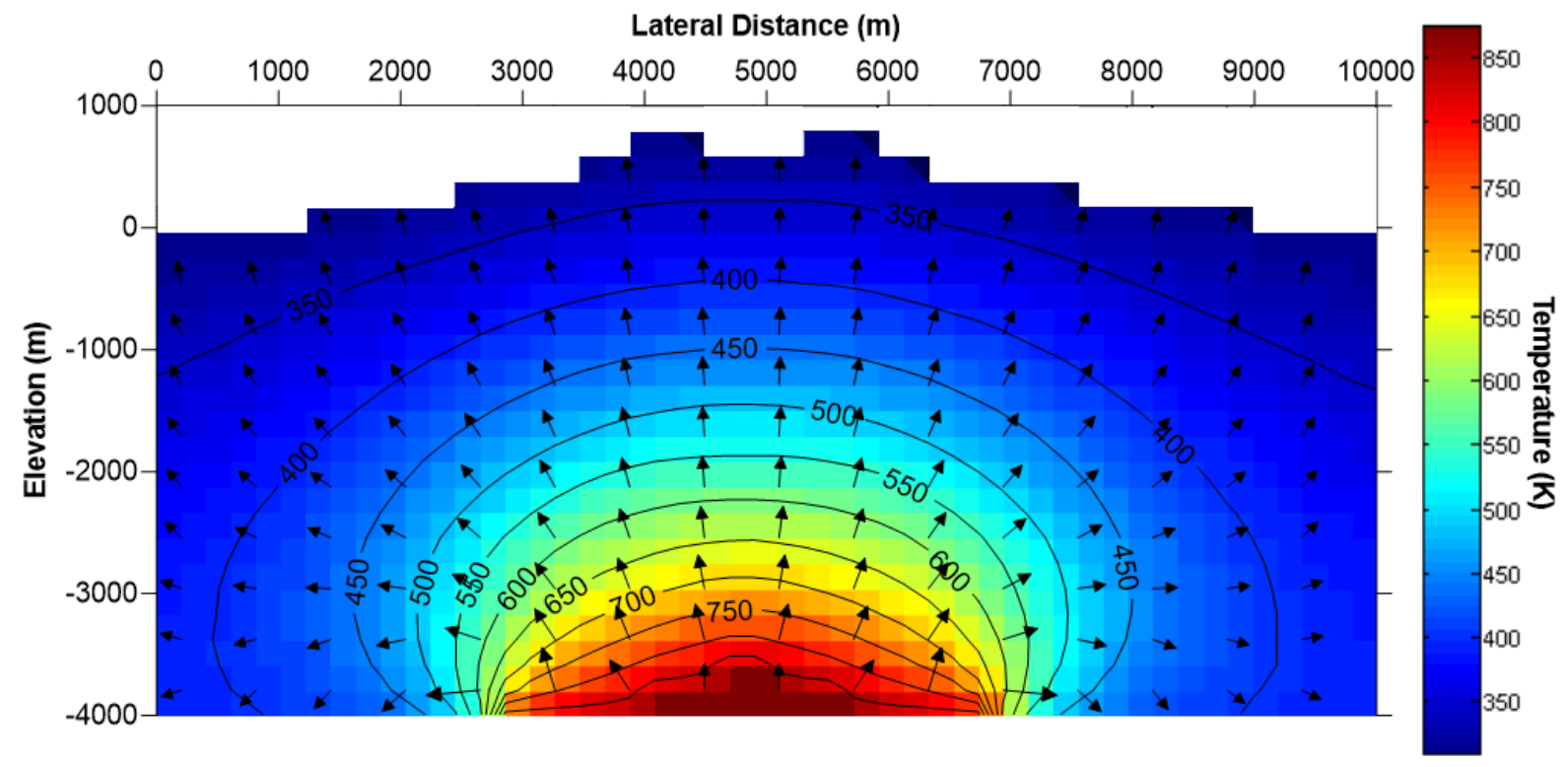

(B)

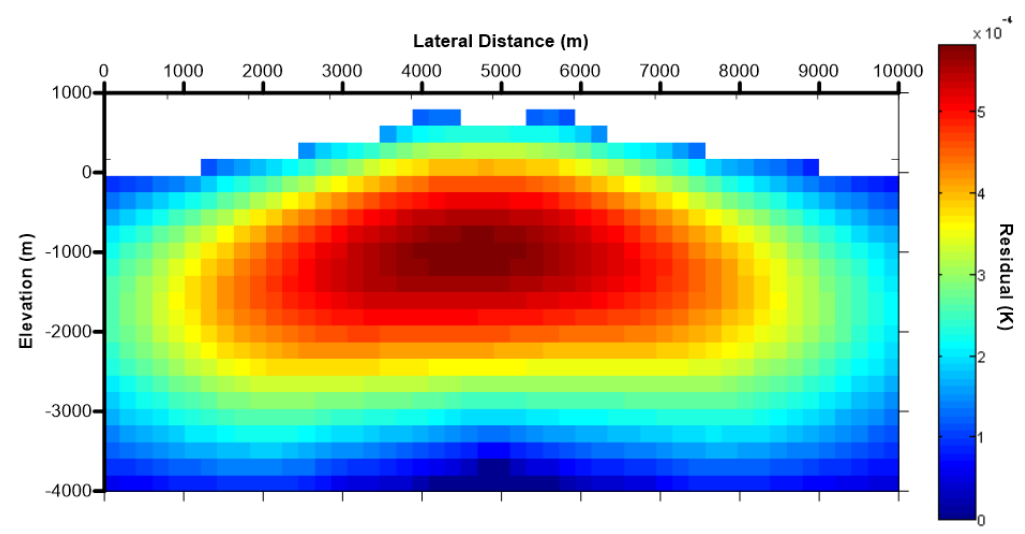

(C)

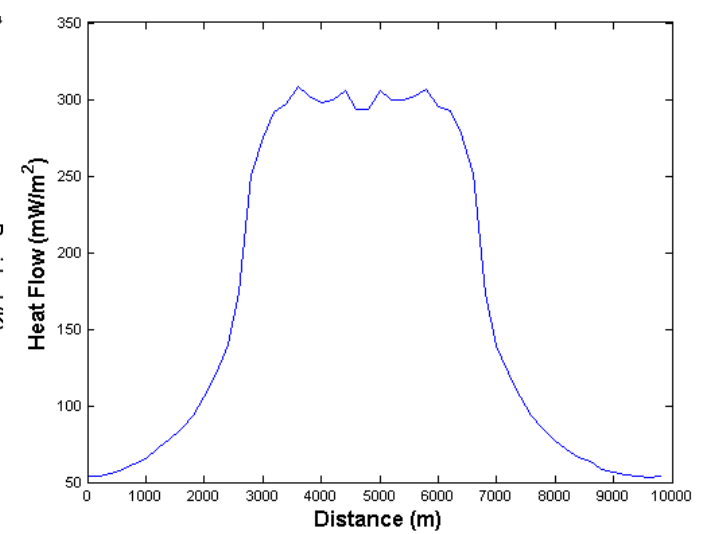

Figure 7. The modeled temperature (A), distribution of residuals (B), and surface heat flow profile (C) from the result of numerical modeling of the volcanic Model 1. Arrows indicate the magnitude and direction of heat flux vectors. 
(A)

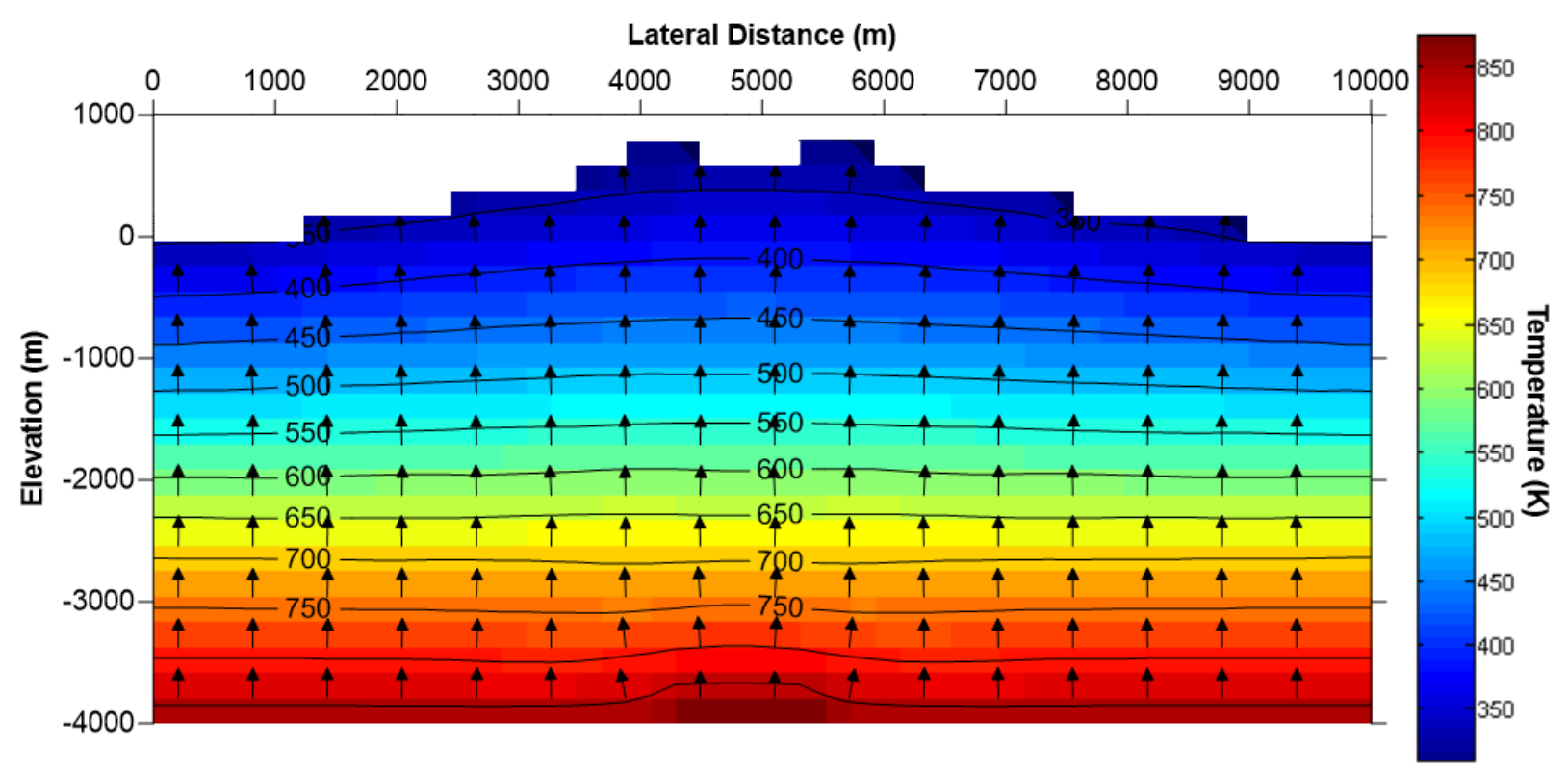

(B)

(C)
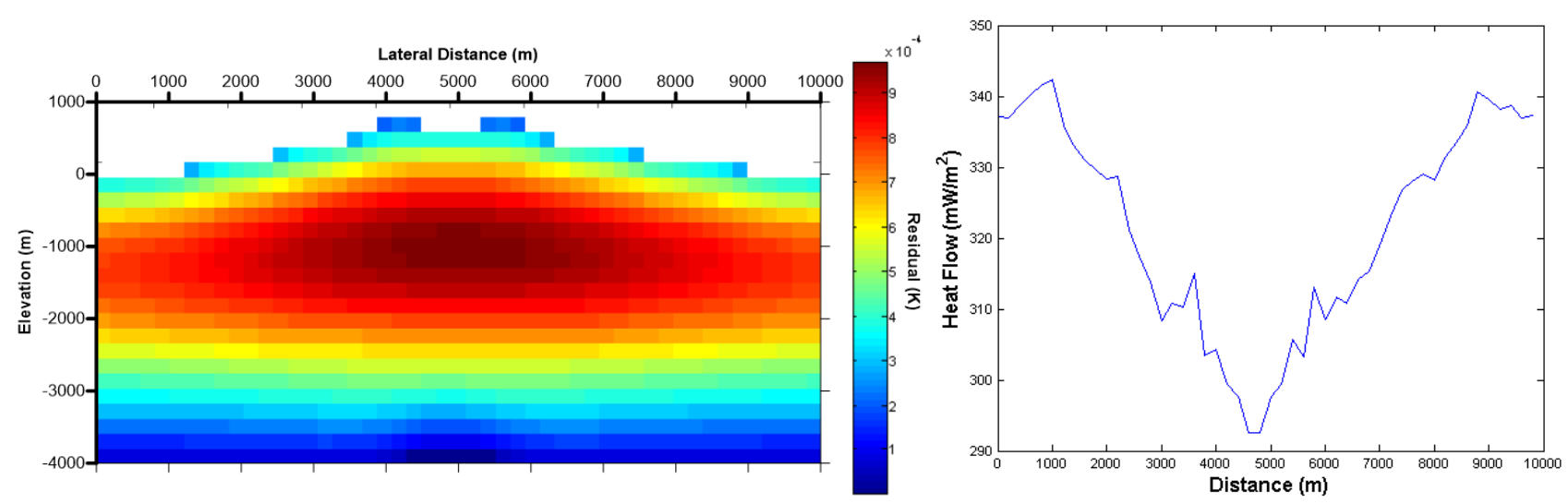

Figure 8. The modeled temperature (A), distribution of residuals (B), and surface heat flow profile (C) from the result of numerical modeling of the volcanic Model 2. Arrows indicate the magnitude and direction heat flux vectors.

The numerical modeling result for the sedimentary basin Model 1 and Model 2 can be seen in Figure 9 and Figure 10. Comparison between the two models clearly provides yet another confirmation on the relative influence of different parameters on the subsurface thermal characteristics of a particular geothermal setting. The relatively flat topography and vertically layered thermal conductivity structure reduces the effect of both parameters on the modeled temperature structure. Contrary to the former synthetic case, the pattern of observed heat flow profile in both sedimentary basin cases does not display a significant difference, except for the absolute magnitude due to the difference in the depth to $200{ }^{\circ} \mathrm{C}$ constant temperature layer. This fact implies that in the absence of topography, the only factor that matters to the observed heat flow anomaly is the subsurface distribution of rocks and their corresponding thermal conductivity values.

\section{CONCLUSIONS}

We have performed a numerical forward modeling procedure of the conductive subsurface temperature distribution by the use of finite difference approximation of the 2-D steady state heat conduction equation via a GaussSeidel linear solver algorithm. The algorithm was capable of resolving the temperature distribution of the synthetic geological models well while maintaining its simplicity, with the number of required iterations for achieving residual values in the order of as low as $10^{-4}$ being 200 iterations. 
(A)

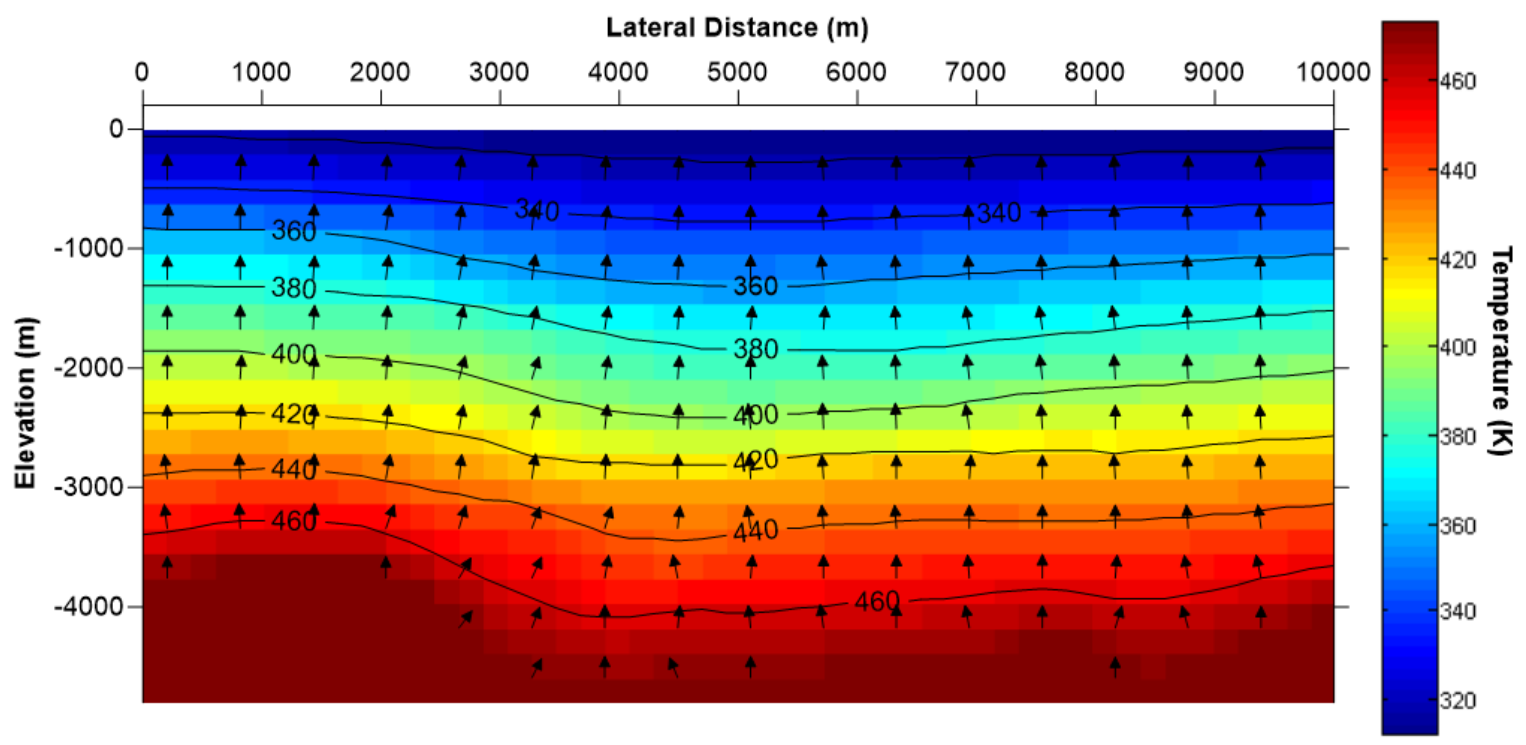

(B)

(C)
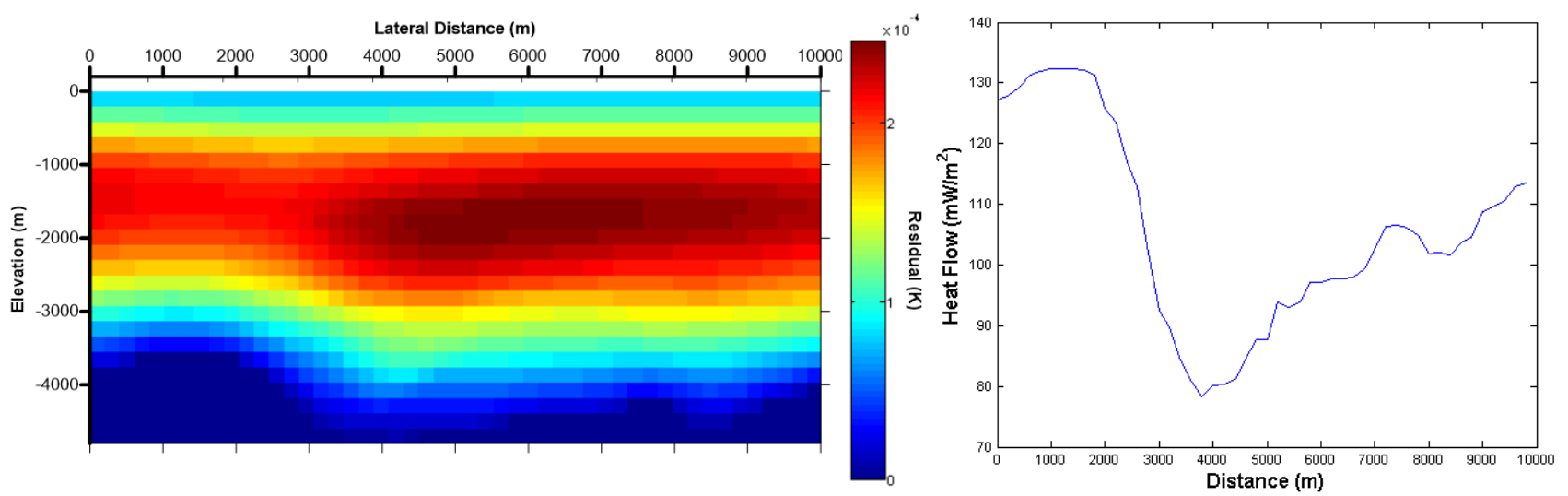

Figure 9. The modeled temperature (A), distribution of residuals (B), and surface heat flow profile (C) from the result of numerical modeling of the sedimentary basin Model 1 . Arrows indicate the magnitude and direction heat flux vectors.

The modeling results using synthetic cases of two common environments of geothermal systems, which are the volcanic and sedimentary basin-hosted settings, were able to explain possible thermal characteristics and relationships between the thermal parameters associated with each system, namely the thermal conductivity, temperature distribution, heat source geometry, and surface topography.

From the above discussions it may therefore be legitimate to deduce that theoretically, for a volcanic hosted geothermal system, the geometry of the magmatic heat source should be the primary control on the overall subsurface temperature distribution, while surface topography and subsurface rock thermal conductivity structure constitute a more important role in determining the observed surface heat flow pattern. The broader the lateral extent of the magmatic heat source, the more topographical and thermal conductivity factors will overwhelm the geometry of the causative heat source in defining the shape of the surface heat flow anomaly, and vice-versa. 
(A)

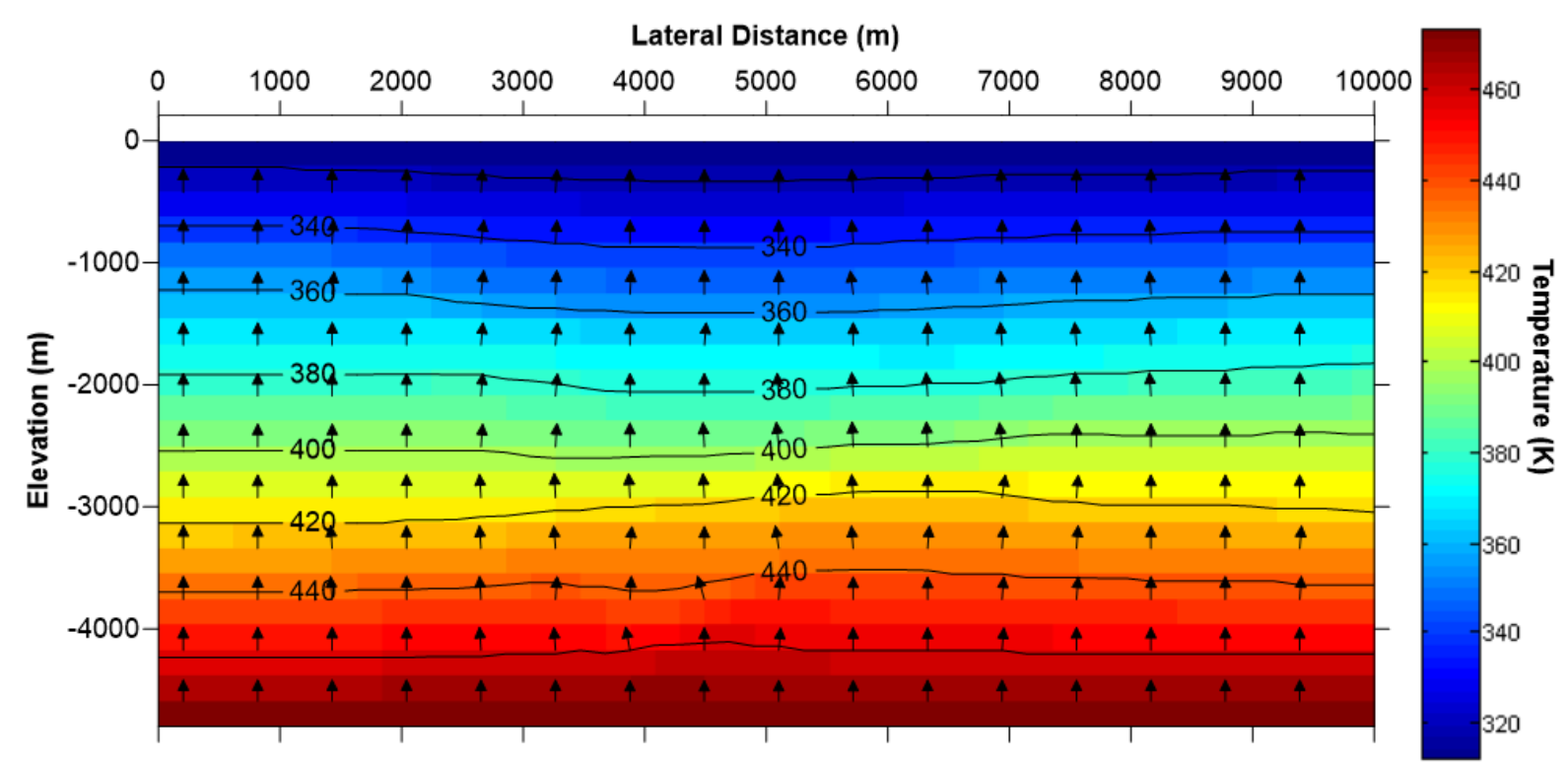

(B)

(C)
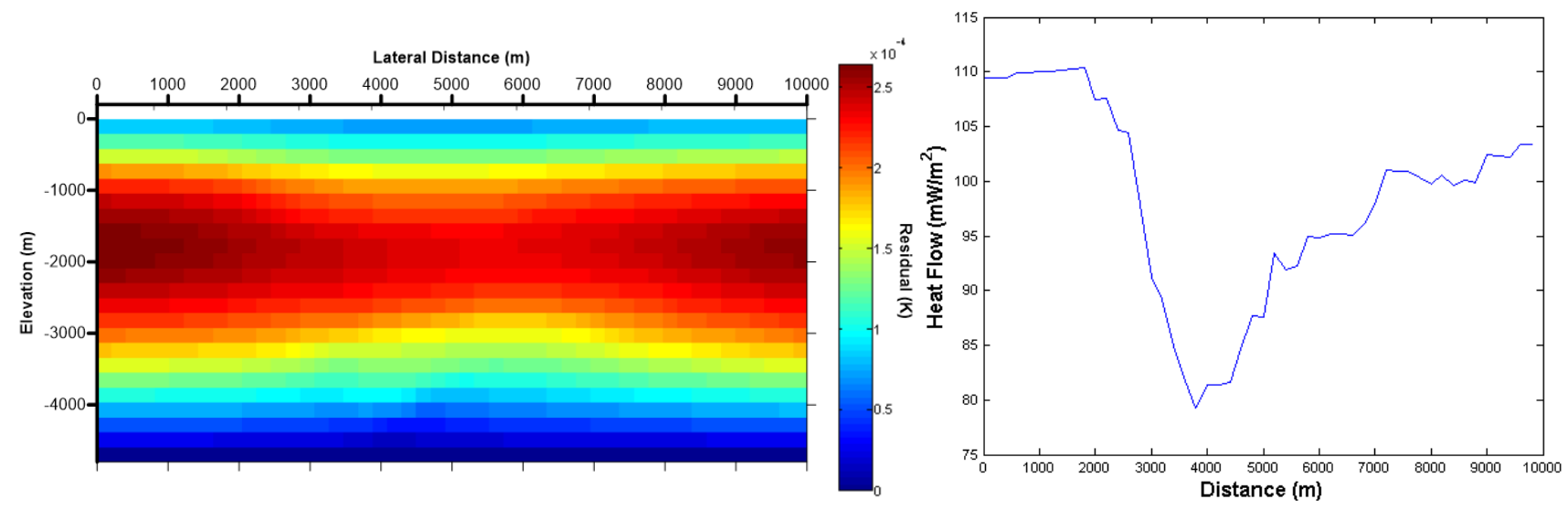

Figure 10. The modeled temperature (A), distribution of residuals (B), and surface heat flow profile (C) from the result of numerical modeling of the sedimentary basin Model 2. Arrows indicate the magnitude and direction heat flux vectors.

As for the sedimentary basin setting, while the subsurface temperature distribution is still mainly controlled by the geometry of the constant temperature layer, the same is not true for the observed surface heat flow. The geometry, depths, and lateral extent of the constant temperature layer do not significantly alter the pattern of surface heat flow due to the absence of undulating topography. Instead, thermal conductivity structure becomes the dominant factor in this particular kind of geothermal environment. Nevertheless, the presence of thick insulating sedimentary layers at the central part of the basin is represented by tighter contour line spacing in that particular zone than in adjacent areas. This suggests that the numerical modeling results were able to resolve the existence of a thermal conductivity anomaly within the basin, i.e. thick low-conductivity sediments overlying a deep, highly conductive granitic basement.

The work has an important implication to the future thermal characterization of geothermal systems in that it will become possible to predict subsurface thermal characteristics by means of a simple numerical simulation to locate possible zones of economically high temperature at depths as well as to estimate the gross stored thermal energy for future development plans using data derived from shallow, inexpensive heat flow measurements. However, further 
studies involving numerical modeling of three-dimensional coupled conductive-convective heat transfer as well as comparative analyses using additional cases from other types of geothermal environments may become a necessity for a more rigorous and detailed investigation into the thermal behaviors of these systems.

\section{ACKNOWLEDGEMENT}

The authors thank the Geothermal Laboratory of the Postgraduate Program of Geothermal Engineering, Faculty of Mining and Petroleum Enginnering for their support by kindly providing the PC workstation necessary for performing all computations in this research.

\section{REFERENCES}

[1] G.R. Beardsmore, J.P. Cull, Crustal Heat Flow: A Guide to Measurement and Modelling. New York, NY: Cambridge University Press, 2001.

[2] G. Buntebarth, Geothermics: An Introduction. Berlin: Springer-Verlag, 1984.

[3] M. Cacace, B.O. Kaiser, B. Lewerenz, M. ScheckWenderoth, "Geothermal energy in sedimentary basins: what we can learn from numerical models," Chemie der Erde vol. 70 S3, May 2010, pp. 33-46, doi:10.1016/j.chemer.2010.05.017.

[4] Y. Cengel, A.J. Ghajar, Heat and Mass Transfer: Fundamentals and Applications $4^{\text {th }}$ Edition in SI Units. New York, NY: McGraw-Hill Higher Education, 2011.

[5] S. Chapra, Applied Numerical Methods with MATLAB for Engineers and Scientists. New York, NY: McGraw-Hill Science/Engineering/Math, 2011.

[6] A. Correia, J. Safanda, "Geothermal modeling along a twodimensional crustal profile in Southern Portugal," Journal of Geodynamics vol 34, Dec. 2001, pp. 47-61, PII: S02643707(01)00080-1.

[7] D. Deming, Introduction to Hydrogeology. New York, NY: McGraw-Hill Science/Engineering/Math, 2011.
[8] H. Gibson, K. Stuwe, R. Seikel, D. FitzGerald, P. Calcagno, A. Guillen, P. McInerney, "Forward prediction of temperature distribution direct from 3D geology models", Australian Geothermal Energy Conference 2008.

[9] F. Goff, C.J. Janik, "Geothermal systems," in Encyclopedia of Volcanoes, H. Sigurdsson, (Ed). Academic Press, 2000, pp. 817-835.

[10] H.K. Gupta, S. Roy, Geothermal Energy: An Alternative Resource for the $21^{\text {st }}$ Century. Amsterdam: Elsevier, 2007.

[11] R. Haenel, L. Rybach, L. Stegena, "Fundamentals of geothermics," in Handbook of Terrestrial Heat Flow Density Determination with Guidelines and Recommendations from The International Heat Flow Commission, R. Haenel, L. Rybach, L. Stegena, (Eds). Dordrecht, Holland: Kluwer Academic Publishers, 1988, pp. 9-56.

[12] M.P. Hochstein, P.R.L. Browne, "Surface manifestations of geothermal systems with volcanic heat sources," in Encyclopedia of Volcanoes, H. Sigurdsson, (Ed). Academic Press, 2000, pp. 835-857.

[13] C. Jaupart, J.C. Mareschal, Heat Generation and Transport in the Earth. New York, NY: Cambridge University Press, 2011.

[14] Kutas, R., Kobolev, V., Korchagin, I., Rusakov, O., Zubal, S., "A software for thermal modeling and its implications for the East-European Craton," Proc. World Geothermal Congress, Apr. 2005, Antalya, Turkey.

[15] S. Nagihara,"Three dimensional inverse modeling of refractive heat flow anomaly associated with salt diapirism," AAPG Bulletin, vol 87 no 7, pp. 1207-1222, July 2003.

[16] J. Safanda, "Calculation of temperature distribution in twodimensional geothermal profile," Studia geoph. et geod. 29, 1985, pp. 197-207.

[17] K. Stuwe, Geodynamics of the Lithosphere: An Introduction. Berlin: Springer, 2002.

[18] K. Stuwe, "Principles of heat flow modeling," Course Notes on Heat Flow for INTREPID GEOPHYSICS, Mar. 2008.

[19] K. Pruess, T.N. Narasimhan, "A practical method for modeling fluid and heat flow in fractured porous media," Society of Petroleum Engineers Reservoir Simulation Symposium, Jun. 1984, New Orleans. 